Article

\title{
Trans-Selective Rhodium Catalysed Conjugate Addition of Organoboron Reagents to Dihydropyranones
}

\author{
Hannah J. Edwards, Sean Goggins and Christopher G. Frost * \\ Department of Chemistry, University of Bath, Claverton Down, Bath BA2 7AY, UK; \\ E-Mails: han.j.edwards@gmail.com (H.J.E.); sg297@bath.ac.uk (S.G.) \\ * Author to whom correspondence should be addressed; E-Mail: c.g.frost@bath.ac.uk; \\ Tel.: +44-(0)1225-386142; Fax: +44-(0)1225-386231.
}

Academic Editor: John Spencer

Received: 10 March 2015 / Accepted: 1 April 2015 / Published: 8 April 2015

\begin{abstract}
The selective synthesis of 2,6-trans-tetrahydropyran derivatives employing the rhodium catalysed addition of organoboron reagents to dihydropyranone templates, derived from a zinc-catalysed hetero-Diels-Alder reaction, is reported. The addition of both arylboronic acids and potassium alkenyltrifluoroborates have been accomplished in high yields using commercially-available $[\mathrm{Rh}(\mathrm{cod})(\mathrm{OH})]_{2}$ catalyst. The selective formation of the 2,6-trans-tetrahydropyran stereoisomer is consistent with a mechanism involving alkene association and carbometalation on the less hindered face of the dihydropyranone.
\end{abstract}

Keywords: boronic acids; conjugate addition; rhodium; tetrahydropyran

\section{Introduction}

The rhodium-catalysed conjugate addition of organometallic donors has evolved into a versatile tool for the assembly of complex molecules and intermediates in natural product synthesis [1-6]. The mechanistic and stereochemical aspects of the reaction have been thoroughly investigated for additions to prochiral substrates [7] and processes involving enantioselective protonation [8-10]. When the addition occurs to a chiral acceptor, the diastereoselectivity can be controlled by substrate [11], ligand [12] or organometallic donor [13]. Tetrahydropyran (THP) rings are a prevalent feature in natural products and such compounds frequently have important biological activities (Figure 1). In this context, the selective assembly of the 2,6-trans-tetrahydropyran subunit is a significant challenge [14]. 
A powerful methodology for the construction of 6-membered heterocycles is the hetero-Diels-Alder (HDA) cycloaddition [15]. This has been a key reaction for the synthesis of many THP containing natural products $[16,17]$. In this paper, we describe a general selective synthesis of 2,6-trans-tetrahydropyran derivatives employing the rhodium-catalysed addition of organoboron reagents to dihydropyranone templates derived from a HDA reaction.

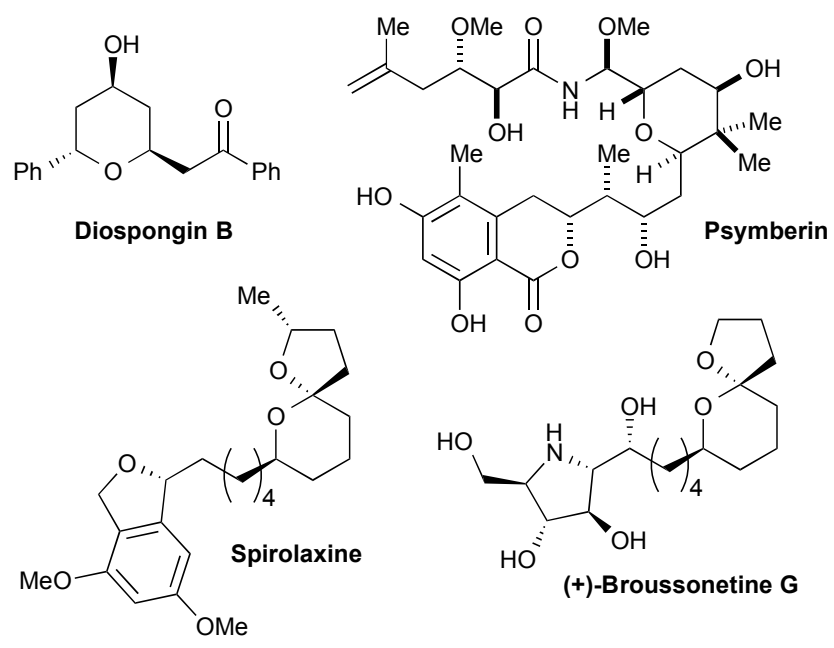

Figure 1. Representative examples of 2,6-trans-tetrahydropyran natural products.

\section{Results and Discussion}

The required 5,6-dihydro- $2 H$-pyranones, can be accessed using a zinc-catalysed HDA reaction of Danishefsky's Diene 1 and an aldehyde heterodienophile [18]. As illustrated in Scheme 1, the use of benzaldehyde 2 results in an efficient synthesis of rac-2-phenyl-2,3-dihydro-pyran-4-one 3 in 98\% isolated yield. Initial investigations into the rhodium-catalysed addition of phenylboronic acid 4 to 3 were carried out using $3 \mathrm{~mol} \%[\mathrm{Rh}(\mathrm{OH})(\mathrm{cod})]_{2}$ with additional ligand in dioxane:water $(10: 1)$ at $80{ }^{\circ} \mathrm{C}$. ${ }^{1} \mathrm{H}-\mathrm{NMR}$ and chiral HPLC analysis of the isolated product indicated the formation of rac-2,6-transdiphenyltetrahydropyran $\mathbf{5}$ in excellent yield.

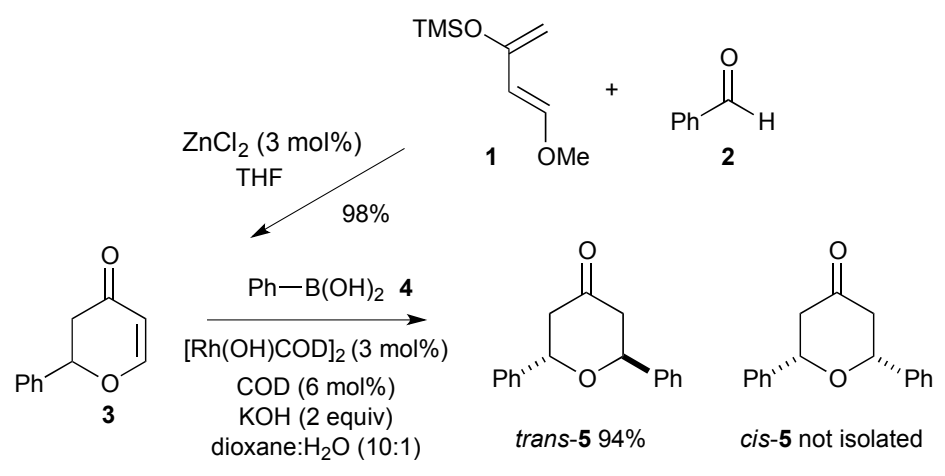

Scheme 1. Catalytic synthesis of 2,6-trans-tetrahydropyran derivatives.

A successful catalytic conjugate addition is dependent on an efficient transmetalation of the organoboronic acid to rhodium followed by carbometallation to afford an $\eta^{3}$-oxa- $\pi$-allylrhodium complex that is protonated to afford the product. A number of detailed mechanistic studies for rhodium-catalysed 
conjugate addition to cyclic and acyclic, activated alkenyl species have been reported [1-6]. The selective formation of the 2,6-trans-tetrahydropyran stereoisomer is consistent with a mechanism involving alkene association and carbometalation on the less hindered face of the dihydropyranone, which affords the 2,6-trans-tetrahydropyran derivative on protonation of the rhodium oxa- $\pi$-allyl species (Figure 2).

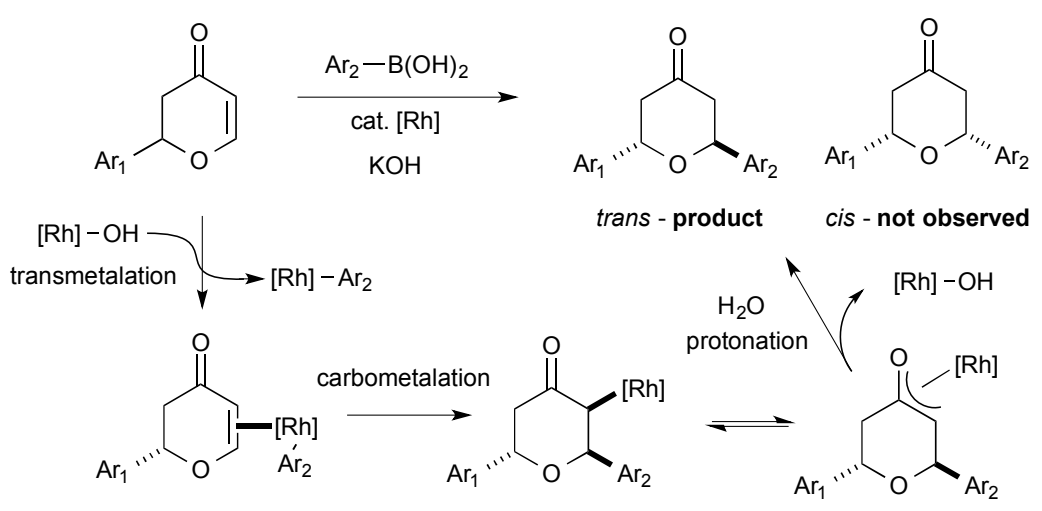

Figure 2. Mechanistic steps and origin of trans-selectivity.

Following these successful initial results, a small range of functionalised 2,3-dihydropyran-4-one substrates were prepared using the zinc-catalysed HDA reaction (Figure 3). It is interesting to note that in many of the 2,6-trans-tetrahydropyran natural products, alkyl chains appear more frequently than aryl groups. Since the use of alkenylboronates in rhodium catalysed additions is well established, this tactical approach presents a synthetic opportunity to install a broad array of functionality from either HDA or conjugate addition. To establish useful scope for synthetic applications it was important to establish whether similar stereocontrol would be maintained in the addition of both aryl- and alkenylboronates.
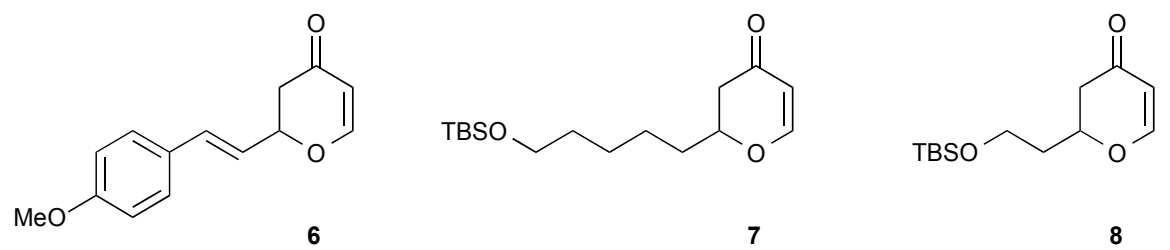

Figure 3. 2,3-Dihydropyran-4-one substrates.

We have previously noted that alkenyltrifluoroborate salts offer practical advantages in terms of stability and product yield in rhodium-catalysed conjugate addition reactions [19]. This is proposed to be due to the slow release of alkenylboronic acid and a concommitant reduction in competing protodeboronation pathways [20]. Therefore, rhodium-catalysed conjugate additions of arylboronic acids and potassium alkenyltrifluoroborates to the 2,3-dihydropyran-4-one substrates were explored. The optimised conditions for the addition of both arylboronic acids and potassium alkenyltrifluoroborates employed commercially-available $[\mathrm{Rh}(\mathrm{cod})(\mathrm{OH})]_{2}$ catalyst with added cycloctadiene ligand to limit catalyst decomposition. A diverse range of organoboronates were shown to successfully participate in the conjugate addition to 2,3-dihydropyran-4-ones affording the products 9-17 as the trans isomer (Scheme 2). 

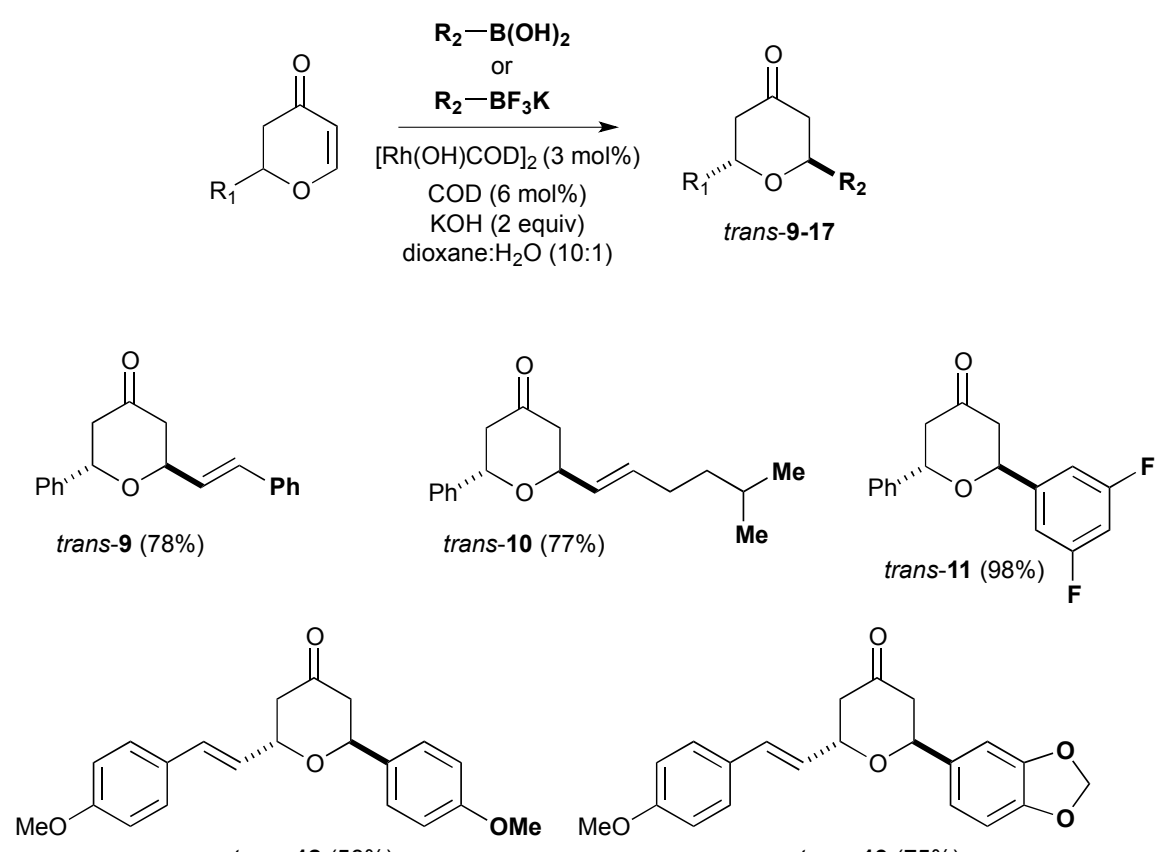

trans-12 (58\%)

trans-13 (75\%)<smiles>CCCC(C)(C)C(C)/C=C/C1CC(=O)C[C@H](/C=C/c2ccc(OC)cc2)O1</smiles><smiles>CCCCOCCCCC[C@H]1CC(=O)C[C@H](c2ccccc2)O1</smiles><smiles>CC(C)OCCCCC[C@@H]1CC(=O)CC(/C=C/c2ccccc2)O1</smiles><smiles>O=C1CC(/C=C/CCOCc2ccccc2)O[C@@H](CCO)C1</smiles>

Scheme 2. Catalytic synthesis of racemic 2,6-trans-tetrahydropyran derivatives.

The synthetic potential of this selective process was next explored with an enantiopure acceptor (Scheme 3). In this context, a suitable asymmetric synthesis of 2-phenyl-2,3-dihydro-pyran-4-one 3 was required. For catalytic asymmetric HDA reactions, a wide-range of chiral Lewis acid complexes have been successfully employed [21]. In particular, the use of $\left.\mathrm{Ti}_{(\mathrm{OPr}}{ }^{i}\right)_{4}$ in combination with $\mathrm{H}_{8}-\mathrm{BINOL}$ offers excellent enantioselectivites and high yields for a wide range of dihydropyrones [22]. Under the reported conditions $(S)-3$ was obtained in $85 \%$ yield and $90 \%$ ee. This would serve as a useful probe for the stereoselectivity of the catalytic conjugate addition and afford enantioenriched products. Pleasingly, the optimised conditions were effective for the addition of both arylboronic acids (products 5, 11 and 19) and potassium alkenyltrifluoroborates (products $9, \mathbf{1 0}$ and 18). No erosion of enantiopurity was observed in the products indicating a highly stereoselective trans addition. The tetrahydropyran derivatives $\mathbf{5}$ and $\mathbf{9}$ were scaled-up and it was possible to recrystallise the products to amplify the ee to $>99 \%$. Confirmation of enantiopurity was established via NMR spectroscopic analysis, by the appearance of only one set of diastereotopic coupling signals in all environments and via chiral HPLC analysis. 


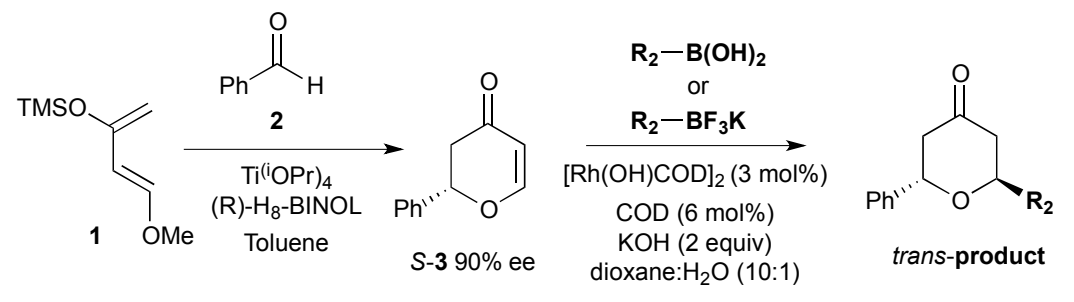

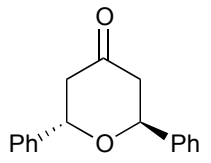

$(S, S)-5(96 \%)$ $90 \%$ ee $(>99 \%$ ee $)$

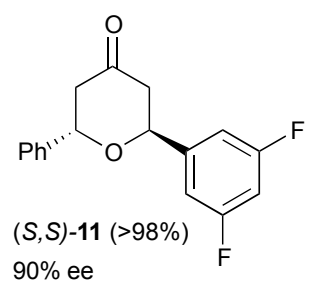

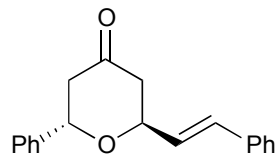

$(S, S)-9(63 \%)$ $90 \%$ ee $(>99 \%$ ee $)$
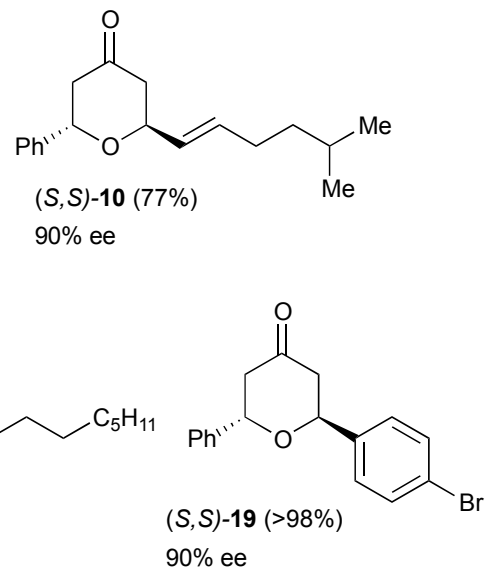

Scheme 3. Catalytic, stereoselective additions to enantioenriched $S$-3.

\section{Experimental Section}

\subsection{General Remarks}

All reactions were carried out under an atmosphere of nitrogen, in oven-dried glassware unless otherwise stated. Dichloromethane, tetrahydrofuran (THF) and toluene were dried and degassed by passing through anhydrous alumina columns using an Innovative Technology Inc. PS-400-7 solvent purification system and stored under an atmosphere of argon prior to use. Proton, carbon, fluorine and phosphorus nuclear magnetic resonance (NMR) spectra were recorded on a Bruker Avance 300 or 400 spectrometer $\left({ }^{1} \mathrm{H}-\mathrm{NMR}\right.$ at 300 or $400 \mathrm{MHz},{ }^{13} \mathrm{C}-\mathrm{NMR}$ at 75.5 or $101 \mathrm{MHz},{ }^{19} \mathrm{~F}-\mathrm{NMR}$ at $376.5 \mathrm{MHz}$ and ${ }^{31} \mathrm{P}-\mathrm{NMR}$ at $121.5 \mathrm{MHz}$ ). Chemical shifts for protons are reported downfield from tetramethylsilane and are referenced to residual protium in the solvent $\left({ }^{1} \mathrm{H}-\mathrm{NMR}: \mathrm{CHCl}_{3}\right.$ at $7.26 \mathrm{ppm}, \mathrm{DMSO}$ at $2.50 \mathrm{ppm}$, $\mathrm{H}_{2} \mathrm{O}$ at $\left.4.79 \mathrm{ppm}\right)$. Chemical shifts for carbons are reported in parts per million downfield from tetramethylsilane and are referenced to the carbon resonances of the solvent peak $\left({ }^{13} \mathrm{C}-\mathrm{NMR}: \mathrm{CDCl}_{3}\right.$ at 77.0 ppm, DMSO- $d_{6}$ at $\left.39.5 \mathrm{ppm}\right)$. IR spectra were recorded on a Perkin-Elmer 1600 FT IR spectrophotometer, with absorbencies quoted as $v$ in $\mathrm{cm}^{-1}$. High resolution mass spectrometry (HRMS) was performed on a $\mu$ TOF electrospray time-of-flight (ESI-TOF) mass spectrometer (Bruker Daltonik). Enantiomeric excesses were determined using HPLC performed on a perkin Elmer IBN series system using chiralcel columns with a UV detector at $254 \mathrm{~nm}$. Melting points were obtained on a Bibby-Sterilin SMP10 melting point machine and are uncorrected.

\subsection{General Procedure for the Synthesis of Racemic Dihydropyranones}

To a flame dried flask under an atmosphere of argon was added $\mathrm{ZnCl}_{2}$ (39 mg, $0.28 \mathrm{mmol}, 3 \mathrm{~mol} \%$ ) and anhydrous diethyl ether $(0.4 \mathrm{~mL}, 3 \mathrm{~mol} \%)$. Anhydrous THF $(100 \mathrm{~mL})$ was added followed by 
freshly-purified aldehyde $(9.42 \mathrm{mmol}, 1.0 \mathrm{eq})$. The reaction was stirred for $10 \mathrm{~min}$ before dropwise addition of Danishefsky's Diene (1) $(2.7 \mathrm{~mL}, 14.13 \mathrm{mmol}, 1.5 \mathrm{eq})$. The reaction was stirred overnight at room temperature and then filtered through celite and concentrated. The crude product was purified by flash column chromatography to afford the respective dihydropyranones.

\subsection{Synthesis of Racemic 2-Phenyl-2,3-dihydropyran-4-one (3)}

Freshly distilled benzaldehyde $(0.96 \mathrm{~mL}, 9.42 \mathrm{mmol})$ was reacted under the standard procedure and the crude product purified by flash column chromatography (eluting with petrol:ethyl acetate $8: 2$ ) to afford the title compound as a red oil ( $1.2 \mathrm{~g}, 73 \%$ yield).

$\mathrm{R}_{\mathrm{f}}$ (petrol:ethyl acetate, 7:3); 0.29; $v_{\max }\left(\mathrm{CH}_{2} \mathrm{Cl}_{2}\right) / \mathrm{cm}^{-1} ; 3063(\mathrm{C}-\mathrm{H}), 1722(\mathrm{C}=\mathrm{O}), 1670(\mathrm{C}=\mathrm{C}), 1593$, $1583(\mathrm{C}=\mathrm{C}), 1268,1037(\mathrm{C}-\mathrm{O}) ; \delta_{\mathrm{H}}\left(300 \mathrm{MHz} ; \mathrm{CDCl}_{3}\right) ; 7.48(1 \mathrm{H}, \mathrm{dd}, J=6.0,0.5 \mathrm{~Hz}, \mathrm{OCH}), 7.44-7.35$ $(5 \mathrm{H}, \mathrm{m}, \mathrm{Ar} H), 5.52(1 \mathrm{H}, \mathrm{dd}, J=6.0 \mathrm{~Hz}, 1.3 \mathrm{~Hz}, \mathrm{CHCO}), 5.43\left(1 \mathrm{H}, \mathrm{dd}, J=14.4,3.5 \mathrm{~Hz}, \mathrm{CH}_{2} \mathrm{CHAr}\right)$, $2.91(1 \mathrm{H}, \mathrm{dd}, J=16.9,14.3 \mathrm{~Hz}, \mathrm{COCHH}), 2.66(\mathrm{ddd}, J=16.9,3.5,1.3 \mathrm{~Hz}, \mathrm{COH} H)$; $\delta_{\mathrm{C}}(75.5 \mathrm{MHz}$; $\left.\mathrm{CDCl}_{3}\right)$; 192.1, 163.2, 137.9, 129.0, 128.9, 126.2, 107.4, 81.1, 43.4; HRMS (ESI ${ }^{+}$) calcd for $\mathrm{C}_{11} \mathrm{H}_{10} \mathrm{NaO}_{2}$ $\left[\mathrm{M}+\mathrm{Na}^{+}\right] \mathrm{m} / \mathrm{z} 197.0579$ found: $m / z$ 197.0590.

All data in accordance with literature values [22].

\subsection{Synthesis of Racemic 2-[2-(4-Methoxyphenyl)vinyl]-2,3-dihydropyran-4-one (6)}

Recrystallised 4-methoxycinnamaldehyde (1.0 g, $6.17 \mathrm{mmol})$ was reacted under the standard procedure and the crude product purified by flash column chromatography (eluting with petrol:ethyl acetate $8: 2)$ to afford the title compound as an orange solid ( $0.28 \mathrm{~g}, 20 \%$ yield).

$\mathrm{R}_{\mathrm{f}}$ (petrol:ethyl acetate, $\left.4: 1\right) ; 0.29 ; \delta_{\mathrm{H}}\left(300 \mathrm{MHz} ; \mathrm{CDCl}_{3}\right) ; 7.40(2 \mathrm{H}, \mathrm{d}, J=6.0 \mathrm{~Hz}, \mathrm{ArH}), 7.34(1 \mathrm{H}, \mathrm{d}$, $J=8.5 \mathrm{~Hz}, \mathrm{OCHCH}), 6.87(2 \mathrm{H}, \mathrm{d}, J=8.5 \mathrm{~Hz}, \operatorname{Ar} H), 6.70(1 \mathrm{H}, \mathrm{d}, J 15.9 \mathrm{~Hz}, \operatorname{ArCHCH}), 6.16(1 \mathrm{H}, \mathrm{dd}$, $J=15.9,6.8 \mathrm{~Hz}, \operatorname{ArCHCH}), 5.46(1 \mathrm{H}, \mathrm{d}, J=6.0 \mathrm{~Hz}, \mathrm{COCH}), 5.07-5.00\left(1 \mathrm{H}, \mathrm{m}, \mathrm{CH}_{2} \mathrm{CHO}\right), 3.81(3 \mathrm{H}$, s, $\left.\mathrm{OCH}_{3}\right), 2.78-2.56\left(2 \mathrm{H}, \mathrm{m}, \mathrm{CH}_{2} \mathrm{CO}\right) ; \delta_{\mathrm{C}}\left(75.5 \mathrm{MHz} ; \mathrm{CDCl}_{3}\right) ; 192.2,163.2,150.0,133.6,128.2,128.1$, 122.7, 114.1, 107.3, 80.1, 55.4, 42.1; HRMS $\left(\mathrm{CI}^{+}\right)$calcd for $\mathrm{C}_{14} \mathrm{H}_{15} \mathrm{O}_{3}[\mathrm{M}+\mathrm{H}]^{+} / z 231.1016$ found: $\mathrm{m} / \mathrm{z} .231 .1059$.

All data in accordance with literature values [22].

\subsection{Synthesis of Racemic 2-[5-(tert-Butyldimethylsilanyloxy)pentyl]-2,3-dihydropyran-4-one (7)}

6-(tert-Butyldimethylsilanyloxy)hexanal $(1.0 \mathrm{~g}, 4.09 \mathrm{mmol})$ was reacted under the standard procedure and the crude product purified by flash column chromatography (eluting with petrol:ethyl acetate $9: 1)$ to afford the title compound as a yellow oil ( $0.990 \mathrm{~g}, 81 \%$ yield).

$\mathrm{R}_{\mathrm{f}}$ (petrol:ethyl acetate, 4:1); 0.56; $\delta_{\mathrm{H}}\left(300 \mathrm{MHz} ; \mathrm{CDCl}_{3}\right) ; 7.35(1 \mathrm{H}, \mathrm{d}, J=5.9 \mathrm{~Hz}, \mathrm{CHCHO}), 5.39(1 \mathrm{H}$, $\mathrm{dd}, J=5.9,1.0 \mathrm{~Hz}, \mathrm{CHCHO}), 4.39\left(1 \mathrm{H}, \mathrm{ddt}, J=12.7,7.7,4.6 \mathrm{~Hz}, \mathrm{CH}_{2} \mathrm{CHCH}_{2}\right), 3.61(2 \mathrm{H}, \mathrm{t}, J=6.3 \mathrm{~Hz}$, $\left.\mathrm{CH}_{2} \mathrm{OSi}\right), 2.52(1 \mathrm{H}, \mathrm{dd}, J=16.7,12.9 \mathrm{~Hz}, \mathrm{COCHH}), 2.42(1 \mathrm{H}, \mathrm{ddd}, J=16.7,4.3,1.0 \mathrm{~Hz}, \mathrm{COCH} H)$, 1.89-1.64 (2H, m, $\left.\mathrm{CHCH}_{2} \mathrm{CH}_{2}\right), 1.58-1.49\left(2 \mathrm{H}, \mathrm{m}, \mathrm{CH}_{2} \mathrm{CH}_{2} \mathrm{OSi}\right), 1.48-1.33\left(4 \mathrm{H}, \mathrm{m},\left(\mathrm{CH}_{2}\right)_{2}\left(\mathrm{CH}_{2}\right)_{2} \mathrm{OSi}\right)$, 
0.89 (9H, s, $\left.\mathrm{SiC}\left(\mathrm{CH}_{3}\right)_{3}\right), 0.04\left(6 \mathrm{H}, \mathrm{s}, \mathrm{Si}\left(\mathrm{CH}_{3}\right)_{2}\right) ; \delta_{\mathrm{c}}\left(75.5 \mathrm{MHz} ; \mathrm{CDCl}_{3}\right) ; 192.9,163.4,107.0,79.6,63.0$, 41.9, 34.5, 32.7, 26.0, 25.7, 24.7, 18.4, -5.2; HRMS $\left(\mathrm{ESI}^{+}\right)$calcd for $\mathrm{C}_{16} \mathrm{H}_{30} \mathrm{O} 3 \mathrm{Si}[\mathrm{M}+\mathrm{H}]^{+} \mathrm{m} / z 299.2037$ found: $m / z 299.2052$.

All data in accordance with literature values [23].

3.6. Synthesis of Racemic 2-[2-(tert-Butyl-dimethyl-silanyloxy)-ethyl]-2,3-dihydropyran-4-one (8)

3-(tert-butyldimethylsilanyloxy)propionaldehyde $(1.0 \mathrm{~g}, 9.336 \mathrm{mmol})$ was reacted under the standard procedure and the crude product purified by flash column chromatography (eluting with petrol:ethyl acetate $4: 1)$ to afford the title compound as a yellow oil ( $0.400 \mathrm{~g}, 39 \%$ yield).

$\mathrm{R}_{\mathrm{f}}$ (petrol:ethyl acetate, 4:1); 0.51; $\delta_{\mathrm{H}}\left(300 \mathrm{MHz} ; \mathrm{CDCl}_{3}\right) ; 7.35(1 \mathrm{H}, \mathrm{d}, J=6.0 \mathrm{~Hz}, \mathrm{OCH}), 5.41(1 \mathrm{H}, \mathrm{dd}$, $J=6.0,1.0 \mathrm{~Hz}, \mathrm{CHCO}), 4.67-4.57\left(1 \mathrm{H}, \mathrm{m}, \mathrm{OCHCH}_{2}\right), 3.85-3.71\left(2 \mathrm{H}, \mathrm{m}, \mathrm{OCH}_{2}\right), 2.63-2.43(2 \mathrm{H}, \mathrm{m}$, $\left.\mathrm{COCH}_{2}\right), 2.07-1.96(1 \mathrm{H}, \mathrm{m}, \mathrm{CHCHHCH} 2), 1.90-1.79\left(1 \mathrm{H}, \mathrm{m}, \mathrm{CHCH} H \mathrm{CH}_{2}\right), 0.89\left(9 \mathrm{H}, \mathrm{s}, \mathrm{SiC}\left(\mathrm{CH}_{3}\right)_{3}\right)$, 0.05 (6H, s, $\left.\mathrm{Si}\left(\mathrm{CH}_{3}\right)_{2}\right) ; \delta_{\mathrm{c}}\left(75.5 \mathrm{MHz} ; \mathrm{CDCl}_{3}\right) ; 192.7,163.2,107.2,58.4,42.1,37.4,26.0,18.4,-5.32$, -5.36; HRMS (ESI ${ }^{+}$) calcd for $\mathrm{C}_{13} \mathrm{H}_{25} \mathrm{O}_{3}[\mathrm{M}+\mathrm{H}]^{+} m / z 257.1572$ found: $m / z 257.1536$.

All data in accordance with literature values [23].

\subsection{Synthesis of (S)-2-Phenyl-2,3-dihydropyran-4-one ((S)-3)}

A mixture of $(R)-\mathrm{H} 8-\mathrm{BINOL}(0.610 \mathrm{~g}, 2.07 \mathrm{mmol})$ and $\mathrm{Ti}\left(\mathrm{O}^{\mathrm{i}} \mathrm{Pr}\right)_{4}(0.56 \mathrm{~mL}, 1.884 \mathrm{mmol})$ with activated $4 \AA$ molecular sieves $(4.54 \mathrm{~g})$ in anhydrous toluene $(38 \mathrm{~mL})$ under an inert atmosphere was heated at $35{ }^{\circ} \mathrm{C}$ for $1 \mathrm{~h}$. The yellow mixture was cooled to room temperature and freshly distilled benzaldehyde $(0.96 \mathrm{~mL}, 9.42 \mathrm{mmol}, 1.0 \mathrm{eq})$ added. After stirring for $10 \mathrm{~min}$ the mixture was cooled to $0{ }^{\circ} \mathrm{C}$ and Danishefsky's diene $(11.3 \mathrm{mmol}, 1.2 \mathrm{eq})$ was added. The reaction was stirred at $0{ }^{\circ} \mathrm{C}$ for $24 \mathrm{~h}$ and then treated with trifluoroacetic acid $(0.1 \mathrm{~mL})$. After stirring for a further $15 \mathrm{~min}$ at $0{ }^{\circ} \mathrm{C}, \mathrm{NaHCO}_{3}$ $(10 \mathrm{~mL})$ was added and the reaction stirred for $10 \mathrm{~min}$ and then filtered through a plug of celite. The organic layer was then separated and the aqueous extracted with diethylether $(3 \times 25 \mathrm{~mL})$. The combined organic extracts were dried over $\mathrm{Na}_{2} \mathrm{SO}_{4}$ and concentrated in vacuo. The crude product was purified by flash chromatography (eluting with petrol:ethyl acetate 8:2) to afford the title compound as a red oil (1.2 $\mathrm{g}$, $73 \%$ yield). The chromatographed material was determined to be in $90 \%$ ee by chiral HPLC analysis (Chiralcel OD, 9:1 Hexanes: propan-2-ol, $1.0 \mathrm{~mL} \cdot \mathrm{min}^{-1}, \mathrm{t}_{\mathrm{R}}=11.22 \mathrm{~min}$ (major) and $13.23 \mathrm{~min}$ (minor).

$\mathrm{R}_{\mathrm{f}}$ (petrol:ethyl acetate, $\left.7: 3\right) ; 0.29 ;[\alpha]_{D}^{20}=+81^{\circ}\left(c=0.8, \mathrm{CHCl}_{3}\right) ; v_{\max }\left(\mathrm{CH}_{2} \mathrm{Cl}_{2}\right) / \mathrm{cm}^{-1} ; 3063(\mathrm{C}-\mathrm{H}), 1722$ $(\mathrm{C}=\mathrm{O}), 1670(\mathrm{C}=\mathrm{C}), 1593,1583(\mathrm{C}=\mathrm{C}), 1268,1037(\mathrm{C}-\mathrm{O}) \delta_{\mathrm{H}}\left(300 \mathrm{MHz} ; \mathrm{CDCl}_{3}\right) ; 7.48(1 \mathrm{H}, \mathrm{dd}, J=6.0$, $0.6 \mathrm{~Hz}, \mathrm{OCHCH}), 7.44-7.38(5 \mathrm{H}, \mathrm{m}, \mathrm{Ar} H), 5.53(1 \mathrm{H}, \mathrm{dd}, J=6.0 \mathrm{~Hz}, 1.2 \mathrm{~Hz}, \mathrm{CHCO}), 5.43(1 \mathrm{H}, \mathrm{dd}$, $\left.J=14.4,3.5 \mathrm{~Hz}, \mathrm{CH}_{2} \mathrm{CHAr}\right), 2.91(1 \mathrm{H}, \mathrm{dd}, J=17.0,14.4 \mathrm{~Hz}, \mathrm{COCHH}), 2.66$ (ddd, $J=17.0,3.5,1.3$ $\mathrm{Hz}, \mathrm{COH} H) ; \delta_{\mathrm{C}}\left(75.5 \mathrm{MHz} ; \mathrm{CDCl}_{3}\right) ; 192.2,163.2,137.9,129.0,128.9,126.2$, 107.5, 81.2, 43.5; HRMS $\left(\mathrm{ESI}^{+}\right)$calcd for $\mathrm{C}_{11} \mathrm{H}_{10} \mathrm{NaO}_{2}[\mathrm{M}+\mathrm{Na}]^{+} m / z 197.0579$ found: $m / z 197.0590$.

All data in accordance with literature values [22]. 


\subsection{General Procedure for the Rhodium-Catalysed Conjugate Additions to Dihydropyranones}

An oven dried, $24 \mathrm{~mL}$ screw-capped vial equipped with a rubber septum was charged with organoboron reagent $(0.228 \mathrm{mmol}, 2.0 \mathrm{eq}),[\mathrm{Rh}(\mathrm{OH})(\mathrm{cod})]_{2}(0.0016 \mathrm{~g}, 0.00342 \mathrm{mmol}, 3 \mathrm{~mol} \%)$, cyclooctadiene $(0.007 \mathrm{~g}, 0.00684 \mathrm{mmol})$ and potassium hydroxide $(0.009 \mathrm{~g}, 0.228 \mathrm{mmol})$. The reaction vessel was purged with argon and dioxane $(0.5 \mathrm{~mL})$ and water $(0.05 \mathrm{~mL})$ were subsequently added by syringe. The red solution was stirred for 15 minutes at room temperature, before the addition of dihydropyranone $(0.114 \mathrm{mmol}, 1.0 \mathrm{eq})$. The reaction was transferred to a preheated hotplate at $80{ }^{\circ} \mathrm{C}$ for $20 \mathrm{~h}$. Upon completion, the crude reaction mixture was taken up in diethyl ether $(5 \mathrm{~mL})$ and filtered through a short plug of silica (elution; diethyl ether) and the solvent removed in vacuo. The crude residue was purified by flash column chromatography on silica gel to afford the desired compounds.

\subsection{Synthesis (2S,6S)-Diphenyltetrahydropyran-4-one (5)}

Phenylboronic acid (0.210 g, $1.72 \mathrm{mmol})$ was treated with $(S)$-2-phenyl-2,3-dihydropyran-4-one ((S)-3) $(0.150 \mathrm{~g}, 0.86 \mathrm{mmol})$ under the standard conditions. The crude residue was purified by flash column chromatography on silica gel (eluting with petrol:ethyl acetate 9:1) to afford the title compound as a white solid ( $0.207 \mathrm{~g}, 96 \%$ yield).

$\mathrm{R}_{\mathrm{f}}$ (petrol:ethyl acetate, $\left.4: 1\right) ; 0.47 ;[\alpha]_{D}^{20}=-16^{\circ}\left(c=1, \mathrm{CHCl}_{3}\right) ; v_{\max }\left(\mathrm{CH}_{2} \mathrm{Cl}_{2}\right) / \mathrm{cm}^{-1} ; 3067,3066,2974$, $2886(\mathrm{C}-\mathrm{H}), 1714(\mathrm{C}=\mathrm{O}), 1601(\mathrm{C}=\mathrm{C}$ aryl $), 1133(\mathrm{C}-\mathrm{O}) ; \delta_{\mathrm{H}}\left(300 \mathrm{MHz} ; \mathrm{CDCl}_{3}\right) ; 7.27-7.26(5 \mathrm{H}, \mathrm{m}, \mathrm{ArH})$, $7.05(2 \mathrm{H}, \mathrm{m}, \mathrm{CHOCH}), 6.87(2 \mathrm{H}, \mathrm{dd}, J=14.6,6.6 \mathrm{~Hz}, \mathrm{CH} H \mathrm{COCHH}), 6.81(2 \mathrm{H}, \mathrm{dd}, J=15.0,5.0 \mathrm{~Hz}$, $\mathrm{C} H \mathrm{HCOCH} H) ; \delta_{\mathrm{C}}\left(75.5 \mathrm{MHz} ; \mathrm{CDCl}_{3}\right) ; 206.8,139.9,128.8,128.2,126.8,73.6,46.4$; HRMS $\left(\mathrm{ESI}^{+}\right)$ calcd for $\mathrm{C}_{17} \mathrm{H}_{16} \mathrm{NaO}_{2}[\mathrm{M}+\mathrm{Na}]^{+} m / z$ 275.1048 found: $m / z$. 275.1029; HPLC (Chiralcel ODH, 97:3 Hexanes:propan-2-ol, $0.5 \mathrm{~mL} \cdot \mathrm{min}^{-1}, \mathrm{t}_{\mathrm{R}}=11.07 \mathrm{~min}$ (major) and $13.11 \mathrm{~min}$ (minor).

\subsection{Synthesis of (2S,6S)-2-Phenyl-6-styryltetrahydropyran-4-one (9)}

Potassium (E)-styryltrifluoroborate $(0.907 \mathrm{~g}, 4.32 \mathrm{mmol})$ was reacted with $(S)$-2-phenyl-2,3dihydropyran-4-one $((S)-3)(0.20 \mathrm{~g}, 1.148 \mathrm{mmol})$ under the standard conditions. The crude residue was purified by flash column chromatography on silica gel (eluting with petrol:ethyl acetate 9:1) to afford the title compound as a white solid $(0.20 \mathrm{~g}, 63 \%$ yield $)$.

$\mathrm{R}_{\mathrm{f}}$ (petrol:ethyl acetate, $\left.4: 1\right) ; 0.5 ;[\alpha]_{D}^{20}=-77^{\circ}\left(c=1, \mathrm{CHCl}_{3}\right), \nu_{\max }$ (neat)/ $\mathrm{cm}^{-1} ; 3035,2979,2882(\mathrm{C}-\mathrm{H})$, $1720(\mathrm{C}=\mathrm{O}), 1658(\mathrm{C}=\mathrm{C}), 1600,1579(\mathrm{C}=\mathrm{C}$ aryl $), 1231,1048(\mathrm{C}-\mathrm{O}) ; \delta \mathrm{H}\left(300 \mathrm{MHz} ; \mathrm{CDCl}_{3}\right) ; 7.34-7.17$ $(10 \mathrm{H}, \mathrm{m}, \mathrm{Ar} H), 6.53(1 \mathrm{H}, \mathrm{dd}, J=16.3,1.4 \mathrm{~Hz}, \mathrm{ArCH}), 6.23(1 \mathrm{H}, \mathrm{dd}, J=16.3,5.0, \operatorname{ArCHCH}), 5.12(1 \mathrm{H}$, $\mathrm{dd}, J=7.4,5.0 \mathrm{~Hz}, \mathrm{ArCHO}), 4.83(1 \mathrm{H}, \mathrm{ddd}, J=10.7,5.2,1.4 \mathrm{~Hz}, \mathrm{CHCHO}), 2.79-2.63$ (4H, m, $\left.\mathrm{CH}_{2} \mathrm{COCH}_{2}\right) ; \delta_{\mathrm{C}}\left(75.5 \mathrm{MHz} ; \mathrm{CDCl}_{3}\right) ; 206.5,140.3,136.0,133.5,128.8,128.7,128.3,128.2,127.8$, 126.7, 126.5, 73.6, 72.9, 47.8, 45.4; HRMS $\left(\mathrm{ESI}^{+}\right)$calcd for $\mathrm{C}_{19} \mathrm{H}_{18} \mathrm{NaO}_{2}[\mathrm{M}+\mathrm{Na}]^{+} \mathrm{m} / z$ 301.1204 found: $\mathrm{m} / z$. 301.1177; HPLC (Chiralcel ODH; 95.5 Hexanes:propan-2-ol, $1.0 \mathrm{~mL} \cdot \mathrm{min}^{-1}, \mathrm{t}_{\mathrm{R}}=13.37 \mathrm{~min}$ (major) and 21.93 min (minor). 


\subsection{Synthesis of (2S, 6S)-2-(5-Methylhex-1-enyl)-6-phenyltetrahydropyran-4-one (10)}

Potassium (E)-trifluoro(5-methyl-hex-1-enyl)borate $(0.047 \mathrm{~g}, 0.23 \mathrm{mmol})$ was reacted with $(S)$-2-phenyl-2,3-dihydropyran-4-one $((S)-3)(0.020 \mathrm{~g}, 0.115 \mathrm{mmol})$ under the standard conditions. The crude residue was purified by flash column chromatography on silica gel (eluting with petrol:ethyl acetate 9:1) to afford the title compound as a yellow oil ( $0.024 \mathrm{~g}, 77 \%$ yield).

$\mathrm{R}_{\mathrm{f}}$ (petrol:ethyl acetate, 4:1); 0.78; $v_{\max }\left(\right.$ neat) $/ \mathrm{cm}^{-1} ; 3068,2955,2870(\mathrm{C}-\mathrm{H}), 1706(\mathrm{C}=\mathrm{O}), 1648(\mathrm{C}=\mathrm{C})$, $1602(\mathrm{C}=\mathrm{C}$ aryl $), 1268,1069(\mathrm{C}-\mathrm{O}) ; \delta_{\mathrm{H}}\left(300 \mathrm{MHz} ; \mathrm{CDCl}_{3}\right) ; 7.38-7.27(5 \mathrm{H}, \mathrm{m}, \mathrm{ArH}), 5.69(1 \mathrm{H}, \mathrm{dtd}$, $\left.J=15.7,6.3,0.9 \mathrm{~Hz}, \mathrm{CH}_{2} \mathrm{CHCH}\right), 5.57\left(1 \mathrm{H}, \mathrm{ddt}, J=15.7,5.0,1.0 \mathrm{~Hz}, \mathrm{CH}_{2} \mathrm{CHCH}\right), 5.11(1 \mathrm{H}, \mathrm{dd}$, $J=7.4,5.3 \mathrm{~Hz}, \mathrm{ArCHO}), 4.70(1 \mathrm{H}, \mathrm{dd}, J=9.7,4.8 \mathrm{~Hz}, \mathrm{CHCHO}), 2.76(1 \mathrm{H}, \mathrm{dd}, J=14.4,5.7$, $\mathrm{CHHCOCHH}), 2.70(2 \mathrm{H}, \mathrm{d}, J=5.4 \mathrm{~Hz}, \mathrm{CH} H \mathrm{COCHH}), 2.60(1 \mathrm{H}, \mathrm{ddd}, J=14.4,4.6,1.0 \mathrm{~Hz}$, $\mathrm{CHHCOCH} H), 2.10-2.03\left(2 \mathrm{H}, \mathrm{m}, \mathrm{CH}_{2} \mathrm{CH}_{2} \mathrm{CH}\right), 1.53\left(1 \mathrm{H}\right.$, nonet, $\left.J=6.6 \mathrm{~Hz},\left(\mathrm{CH}_{3}\right)_{2} \mathrm{CH}\right), 1.29-1.24$ $\left(2 \mathrm{H}, \mathrm{m},\left(\mathrm{CH}_{3}\right)_{2} \mathrm{CHCH}_{2}\right), 0.88\left(6 \mathrm{H}, \mathrm{d}, J=6.6 \mathrm{~Hz},\left(\mathrm{CH}_{3}\right)_{2} \mathrm{CH}\right) ; \delta_{\mathrm{C}}\left(75.5 \mathrm{MHz} ; \mathrm{CDCl}_{3}\right) ; 206.9,140.5,136.1$, 128.1, 126.4, 73.1, 72.9, 48.0, 45.4, 38.1, 30.4, 27.6, 22.5; HPLC (Chiralcel AD; 98:2 Hexanes:propan-2-ol, $1.0 \mathrm{~mL} \cdot \mathrm{min}^{-1}, \mathrm{t}_{\mathrm{R}}=6.85 \mathrm{~min}$ (major) and $15.91 \mathrm{~min}$ (minor).

\subsection{Synthesis of (2S,6S)-2-(3,5-Difluorophenyl)-6-phenyltetrahydropyran-4-one (11)}

3,5-difluorophenylboronic acid $(0.045 \mathrm{~g}, 0.287 \mathrm{mmol})$ was reacted with $(S)$-2-phenyl-2,3dihydropyran-4-one ((S)-3) (0.025 g, $0.144 \mathrm{mmol})$ under the standard conditions. The crude residue was purified by flash column chromatography on silica gel (eluting with petrol:ethyl acetate 9:1) to afford the title compound as a yellow solid $(0.038 \mathrm{~g}, 98 \%$ yield $)$.

$\mathrm{R}_{\mathrm{f}}$ (petrol:ethyl acetate, 4:1); 0.6; $\delta_{\mathrm{H}}\left(300 \mathrm{MHz} ; \mathrm{CDCl}_{3}\right) ; 7.42-7.30(5 \mathrm{H}, \mathrm{m}, \mathrm{ArH}), 6.90(2 \mathrm{H}$, ddd, $J=8.2,2.2,0.7 \mathrm{~Hz}, \mathrm{FCCHCCHCF}), 6.75(1 \mathrm{H}, \mathrm{tt}, J=8.8,2.3 \mathrm{~Hz}, \mathrm{CFCHCF}), 5.34(1 \mathrm{H}, \mathrm{t}, J=5.7 \mathrm{~Hz}$, OCH), $4.97(1 \mathrm{H}, \mathrm{dd}, J=6.82,5.64 \mathrm{~Hz}, \mathrm{OCH}), 2.93(2 \mathrm{H}, \mathrm{ddd}, J=14.5,5.7,0.9 \mathrm{~Hz}, \mathrm{CH} H C O C H H)$, 2.85-2.72 (2H, m, CHHCOCHH); $\delta_{\mathrm{c}}\left(75.5 \mathrm{MHz} ; \mathrm{CDCl}_{3}\right) ; 205.7,144.2,139.2,128.9,128.5,127.0$, 109.7, 109.3, 103.5, 74.2, 72.3, 46.8, 45.8; HPLC (Chiralcel AD: 99:1 Hexanes:propan-2-ol, $0.5 \mathrm{~mL} \cdot \mathrm{min}^{-1}, \mathrm{t}_{\mathrm{R}}=37.30 \mathrm{~min}$ (major) and $48.68 \mathrm{~min}$ (minor).

\subsection{Synthesis of trans-2-(4-Methoxyphenyl)-6-[2-(4-methoxyphenyl)-vinyl]-tetrahydropyran-4-one (12)}

4-methoxyphenylboronic acid (0.026 g, $0.174 \mathrm{mmol})$ was reacted with 2-[2-(4-Methoxyphenyl)vinyl]-2,3-dihydropyran-4-one $(6)(0.020 \mathrm{~g}, 0.0869 \mathrm{mmol})$ under the standard conditions. The crude residue was purified by flash column chromatography on silica gel (eluting with petrol:ethyl acetate 8:2) to afford the title compound as a yellow oil $(0.017 \mathrm{~g}, 58 \%$ yield $)$.

$\mathrm{R}_{\mathrm{f}}$ (petrol:ethyl acetate, 4:1); 0.26; $\delta_{\mathrm{H}}\left(300 \mathrm{MHz} ; \mathrm{CDCl}_{3}\right) ; 7.32(4 \mathrm{H}, \mathrm{dd}, J$ 8.8, $2.2 \mathrm{~Hz}, \mathrm{ArH}, 6.88(4 \mathrm{H}$, $\mathrm{dd}, J=11.6,8.8 \mathrm{~Hz}, \mathrm{ArH}), 6.52(1 \mathrm{H}, \mathrm{d}, J=16.2,5.3 \mathrm{~Hz}, \mathrm{ArCH}), 6.15(1 \mathrm{H}, \mathrm{d}, J=16.2 \mathrm{~Hz}, 5.3 \mathrm{~Hz}$, $\mathrm{ArCHCH}), 5.17(1 \mathrm{H}, \mathrm{dd}, J=7.2,4.9, \mathrm{ArCHO}), 4.81(1 \mathrm{H}, \mathrm{ddd}, J=9.4,5.3,1.4, \mathrm{CHCHO}), 3.81(6 \mathrm{H}, \mathrm{s}$, $\left.\mathrm{OCH}_{3}, \mathrm{OCH}_{3}\right), 2.87-2.63\left(4 \mathrm{H}, \mathrm{m}, \mathrm{CH}_{2} \mathrm{COCH}_{2}\right)$; HRMS $\left(\mathrm{ESI}^{+}\right)$calcd for $\mathrm{C}_{21} \mathrm{H}_{22} \mathrm{NaO}_{4}[\mathrm{M}+\mathrm{Na}]^{+} \mathrm{m} / z$ 361.1416 found: $m / z .361 .1404$. 
Benzo-[1,3]dioxol-5-ylboronic acid $(0.029 \mathrm{~g}, \quad 0.174 \mathrm{mmol}) \quad$ was reacted with 2-[2-(4methoxyphenyl)-vinyl]-2,3-dihydropyran-4-one (6) (0.020 g, $0.0869 \mathrm{mmol})$ under the standard conditions. The crude residue was purified by flash column chromatography on silica gel (eluting with petrol:ethyl acetate $8: 2)$ to afford the title compound as a yellow oil $(0.032 \mathrm{~g}, 75 \%$ yield).

$\mathrm{R}_{\mathrm{f}}$ (petrol:ethyl acetate, 4:1); 0.38; $v_{\max }\left(\right.$ neat) $/ \mathrm{cm}^{-1} ; 2906,2862(\mathrm{C}-\mathrm{H}), 1715(\mathrm{C}=\mathrm{O}), 1641(\mathrm{C}=\mathrm{C}), 1606$, 1577, $1511(\mathrm{C}=\mathrm{C}$ aryl $), 1246,1033(\mathrm{C}-\mathrm{O}) ; \delta_{\mathrm{H}}\left(300 \mathrm{MHz} ; \mathrm{CDCl}_{3}\right) ; 7.33(3 \mathrm{H}, \mathrm{d}, J 8.8 \mathrm{~Hz}, \mathrm{ArH}), 6.89(2 \mathrm{H}$, $\mathrm{d}, J=11.7 \mathrm{~Hz}, \mathrm{Ar} H), 6.82(2 \mathrm{H}, \mathrm{d}, J=11.5 \mathrm{~Hz}, \mathrm{ArH}), 6.53(1 \mathrm{H}, \mathrm{d}, J=16.2 \mathrm{~Hz}, \operatorname{ArCHCH}), 6.14(1 \mathrm{H}, \mathrm{dd}$, $J=16.4,5.5 \mathrm{~Hz}, \mathrm{ArCHCH}), 5.96\left(2 \mathrm{H}, \mathrm{s}, \mathrm{OCH}_{2} \mathrm{O}\right), 5.10(1 \mathrm{H}, \mathrm{dd}, J=7.0,5.4 \mathrm{~Hz}, \mathrm{ArCHO}), 4.84(1 \mathrm{H}$, ddd, $J=10.6,5.3,1.2 \mathrm{~Hz}, \mathrm{CHCHO}), 3.81\left(3 \mathrm{H}, \mathrm{s}, \mathrm{ArOCH}_{3}\right), 2.83-2.65$ (4H, m, $\left.\mathrm{CH}_{2} \mathrm{COCH}_{2}\right)$; HRMS $\left(\mathrm{ESI}^{+}\right.$) calcd for $\mathrm{C}_{21} \mathrm{H}_{20} \mathrm{NaO}_{5}[\mathrm{M}+\mathrm{H}]^{+} m / z 375.1208$ found: $m / z .375 .1192$.

\subsection{Synthesis of trans-2-Dec-1-enyl-6-[2-(4-methoxyphenyl)vinyl]-tetrahydropyran-4-one (14)}

Potassium decenyl trifluoroborate $(0.043 \mathrm{~g}, 0.174 \mathrm{mmol})$ was reated with 2-[2-(4-methoxyphenyl)vinyl]-2,3-dihydropyran-4-one $(6)(0.020 \mathrm{~g}, 0.0869 \mathrm{mmol})$ under the standard conditions. The crude residue was purified by flash column chromatography on silica gel (eluting with petrol:ethyl acetate 8:2) to afford the title compound as a yellow oil ( $0.026 \mathrm{~g}, 81 \%$ yield $)$.

$\mathrm{R}_{\mathrm{f}}$ (petrol:ethyl acetate, 4:1); 0.5; $v_{\max }\left(\mathrm{CH}_{2} \mathrm{Cl}_{2}\right) / \mathrm{cm}^{-1} ; 2925,2855(\mathrm{C}-\mathrm{H}), 1718(\mathrm{C}=\mathrm{O}), 1610,1514(\mathrm{C}=\mathrm{C}$ aryl), $1250(\mathrm{C}-\mathrm{O}) ; \delta$ н $\left(300 \mathrm{MHz} ; \mathrm{CDCl}_{3}\right) ; 7.32(2 \mathrm{H}, \mathrm{d}, J=8.7 \mathrm{~Hz}, \mathrm{Ar} H), 6.85(2 \mathrm{H}, \mathrm{d}, J=8.7 \mathrm{~Hz}, \mathrm{ArH})$, $6.53(1 \mathrm{H}, \mathrm{d}, J=16.1 \mathrm{~Hz}, \mathrm{ArCHCH}), 6.12(1 \mathrm{H}, \mathrm{dd}, J=16.1,5.6 \mathrm{~Hz}, \mathrm{ArCHCH}), 5.71(1 \mathrm{H}, \mathrm{dt}, J=16.0$, $\left.6.6 \mathrm{~Hz}, \mathrm{CH}_{2} \mathrm{CHCH}\right), 5.55\left(1 \mathrm{H}, \mathrm{dd}, J=15.7,5.5 \mathrm{~Hz}, \mathrm{CH}_{2} \mathrm{CHCH}\right), 4.80(1 \mathrm{H}, \mathrm{dd}, J=10.7,5.1 \mathrm{~Hz}, \mathrm{CHO})$, $4.67(1 \mathrm{H}, \mathrm{dd}, J=10.7,5.6 \mathrm{~Hz}, \mathrm{CHO}), 3.08(3 \mathrm{H}, \mathrm{s}, \mathrm{OCH}), 2.69-2.47\left(4 \mathrm{H}, \mathrm{m}, \mathrm{CH}_{2} \mathrm{COCH}_{2}\right), 2.05(2 \mathrm{H}, \mathrm{q}$, $\left.J=6.9 \mathrm{~Hz}, \mathrm{CH}_{2} \mathrm{CHCH}\right), 1.37-1.26\left(12 \mathrm{H}, \mathrm{m}, \mathrm{CH}_{3}\left(\mathrm{CH}_{2}\right)_{6}\right), 0.88\left(3 \mathrm{H}, \mathrm{t}, J=6.6 \mathrm{~Hz}, \mathrm{CH}_{3}\left(\mathrm{CH}_{2}\right)_{6}\right) ; \delta_{\mathrm{C}}(75.5$ $\left.\mathrm{MHz} ; \mathrm{CDCl}_{3}\right)$; 206.7, 159.9, 135.4, 132.4, 128.6, 127.9, 126.0, 125.7, 114.1, 72.6, 72.5, 55.4, 46.3, 32.5, 32.0, 29.5, 29.3, 29.3, 29.0, 22.8, 14.2; $\mathrm{HRMS}\left(\mathrm{ESI}^{+}\right)$calcd for $\mathrm{C}_{24} \mathrm{H}_{35} \mathrm{O}_{3}[\mathrm{M}+\mathrm{H}]^{+} \mathrm{m} / z 371.2586$ found: $\mathrm{m} / \mathrm{z} 371.2588$.

3.16. Synthesis of trans-2-[5-(tert-Butyl-dimethylsilanyloxy)-pentyl]-6-phenyltetrahydropyran-4-one (15)

Phenylboronic acid $(0.021 \mathrm{~g}, 0.168 \mathrm{mmol})$ was reacted with $(S)$-2-[5-(tert-Butyl-dimethylsilanyloxy)pentyl]-2,3-dihydropyran-4-one (7) (0.025 g, $0.0838 \mathrm{mmol})$ under the standard conditions. The crude residue was purified by flash column chromatography on silica gel (eluting with petrol:ethyl acetate 9:1) to afford the title compound as a colourless oil ( $0.020 \mathrm{~g}, 64 \%$ yield).

$\mathrm{R}_{\mathrm{f}}$ (petrol:ethyl acetate, 4:1); 0.72; $\delta_{\mathrm{H}}\left(300 \mathrm{MHz} ; \mathrm{CDCl}_{3}\right) ; 7.41-7.28(5 \mathrm{H}, \mathrm{m}, \mathrm{Ar} H), 5.21(1 \mathrm{H}, \mathrm{t}, J=5.7$ $\mathrm{Hz}, \mathrm{ArCHO}), 3.98-3.90\left(1 \mathrm{H}, \mathrm{m}, \mathrm{OCHCH}_{2}\right), 3.57\left(2 \mathrm{H}, \mathrm{t}, J=1.56 \mathrm{~Hz}, \mathrm{CH}_{2} \mathrm{OSi}\right), 2.88-2.74(2 \mathrm{H}, \mathrm{m}$, $\left.\mathrm{COCH}_{2}\right), 2.57(1 \mathrm{H}, \mathrm{ddd}, J=14.4,4.5,1.1 \mathrm{~Hz}, \mathrm{COCH}), 2.34(1 \mathrm{H}, \mathrm{dd}, J=14.4,7.3,1.1 \mathrm{~Hz}, \mathrm{COCH} H)$, 1.54-1.40 (4H, m, $\left.\mathrm{CH}_{2} \mathrm{CH}_{2}\right), 1.37-1.27\left(4 \mathrm{H}, \mathrm{m}, \mathrm{CH}_{2} \mathrm{CH}_{2}\right), 0.88\left(9 \mathrm{H}, \mathrm{s}, \mathrm{C}\left(\mathrm{CH}_{3}\right)_{3}\right), 0.03\left(6 \mathrm{H}, \mathrm{s}, \mathrm{Si}\left(\mathrm{CH}_{3}\right)_{2}\right)$; $\delta_{\mathrm{C}}\left(75.5 \mathrm{MHz} ; \mathrm{CDCl}_{3}\right) ; 207.3,140.2,128.6,128.0,126.8,63.1,47.2,46.2,34.55,32.72,25.9,25.6,25.1$, $18.4,-5.26$. 
Potassium $(E)$-styryl trifluoroborate $(0.028 \mathrm{~g}, 0.13 \mathrm{mmol})$ was reacted with $(S)$-2-[5-(tert-Butyldimethyl-silanyloxy)-pentyl]-2,3-dihydropyran-4-one (7) (0.020 g, $0.067 \mathrm{mmol})$ under the standard conditions. The crude residue was purified by flash column chromatography on silica gel (eluting with petrol:ethyl acetate 9:1) to afford the title compound as a colourless oil ( $0.010 \mathrm{~g}, 37 \%$ yield).

$\mathrm{R}_{\mathrm{f}}$ (petrol:ethyl acetate, 4:1); 0.62; $v_{\max }\left(\right.$ neat) $/ \mathrm{cm}^{-1} ; 2931,2858(\mathrm{C}-\mathrm{H}), 1713(\mathrm{C}=\mathrm{O}), 1623(\mathrm{C}=\mathrm{C}), 1579$, $1569(\mathrm{C}=\mathrm{C}$ aryl $), 1251,1049(\mathrm{C}-\mathrm{O}) ; \delta_{\mathrm{H}}\left(300 \mathrm{MHz} ; \mathrm{CDCl}_{3}\right) ; 7.40-7.27(5 \mathrm{H}, \mathrm{ArH}), 6.56(1 \mathrm{H}, \mathrm{dd}$, $J=16.1,1.2, \mathrm{ArCH}), 6.23(1 \mathrm{H}, \mathrm{dd}, J=11.2,5.2, \mathrm{ArCHCH}), 4.87(1 \mathrm{H}, \mathrm{dd}, J=9.6,4.5 \mathrm{~Hz}, \mathrm{CHCHO})$, 4.12-4.03 (1H, m, OCHCH 2$), 3.59(2 \mathrm{H}, \mathrm{t}, J=6.4 \mathrm{~Hz}, \mathrm{CH} 2 \mathrm{OTBS}), 2.68(2 \mathrm{H}, \mathrm{qd}, J=14.3,5.4 \mathrm{~Hz}$, $\left.\mathrm{COCH}_{2}\right), 2.49(1 \mathrm{H}, \mathrm{ddd}, J=14.2,4.0,1.2 \mathrm{~Hz}, \mathrm{COCHH}), 2.29(1 \mathrm{H}, \mathrm{dd}, J=13.9,8.2 \mathrm{~Hz}, \mathrm{COCH} H)$, 1.55-1.46 (4H, m, $\left.\mathrm{CH}_{2} \mathrm{CH}_{2}\right), 1.43-1.34\left(4 \mathrm{H}, \mathrm{m}, \mathrm{CH}_{2} \mathrm{CH}_{2}\right), 0.88\left(9 \mathrm{H}, \mathrm{s}, \mathrm{C}\left(\mathrm{CH}_{3}\right)_{3}\right), 0.03\left(6 \mathrm{H}, \mathrm{s}, \mathrm{Si}\left(\mathrm{CH}_{3}\right)_{2}\right)$.

\subsection{Synthesis of trans-2-(4-Benzyloxybut-1-enyl)-6-(2-hydroxyethyl)tetrahydropyran-4-one (17)}

Potassium (E)-(4-(-benzyloxy)but-1-en-1-yl)trifluoroborate $(0.105 \mathrm{~g}, 0.39 \mathrm{mmol})$ was reated with 2-[2-(tert-Butyldimethylsilanyloxy)ethyl]-2,3-dihydropyran-4-one (8) (0.050 g, $0.195 \mathrm{mmol})$ under the standard conditions. The crude residue was treated with TBAF $(0.43 \mathrm{~mL}, 1 \mathrm{M}$ in THF) in THF (2 mL). After stirring for $1 \mathrm{~h}$, a saturated solution of $\mathrm{NH}_{4} \mathrm{Cl}$ was added and the mixture extracted with $\mathrm{Et}_{2} \mathrm{O}$ $(3 \times 10 \mathrm{~mL})$. Combined organic extracts were dried $\left(\mathrm{MgSO}_{4}\right)$ and concentrated in vacuo. The residue was purified by flash column chromatography on silica gel (eluting with $\mathrm{CH}_{2} \mathrm{Cl}_{2}$ :methanol 9:1) to afford the title compound as a yellow oil $(0.048 \mathrm{~g}, 81 \%$ yield $)$.

$\mathrm{R}_{\mathrm{f}}\left(\mathrm{CH}_{2} \mathrm{Cl}_{2}\right.$ :methanol 9:1); 0.46; $v_{\max }$ (neat) $/ \mathrm{cm}^{-1} ; 3342(\mathrm{O}-\mathrm{H}), 2930,2858(\mathrm{C}-\mathrm{H}), 1472,1463(\mathrm{C}=\mathrm{C})$, 1254, 1094 (C-O); $\delta_{\mathrm{H}}\left(300 \mathrm{MHz} ; \mathrm{CDCl}_{3}\right) ; 7.37-7.27(5 \mathrm{H}, \mathrm{m}, \mathrm{ArH}), 5.71(1 \mathrm{H}, \mathrm{dt}, J=15.8,6.4 \mathrm{~Hz}$, $\left.\mathrm{CH}_{2} \mathrm{CHCH}\right), 5.58\left(1 \mathrm{H}, \mathrm{dd}, J=15.8,4.7 \mathrm{~Hz}, \mathrm{CH}_{2} \mathrm{CHCH}\right), 4.75(1 \mathrm{H}, \mathrm{dd}, J=9.7,4.7 \mathrm{~Hz}, \mathrm{CHCHO}), 4.49$ $\left(2 \mathrm{H}, \mathrm{s}, \mathrm{ArCH}_{2} \mathrm{O}\right), 4.26\left(1 \mathrm{H}, \mathrm{m}, \mathrm{OCHCH}_{2}\right), 3.73(1 \mathrm{H}, \mathrm{br} . \mathrm{s}, \mathrm{OH}), 3.50\left(2 \mathrm{H}, \mathrm{t}, J=6.6 \mathrm{~Hz}, \mathrm{OCH}_{2} \mathrm{CH}_{2}\right), 2.67$ $(1 \mathrm{H}, \mathrm{dd}, J=14.5,6.2 \mathrm{~Hz}, \mathrm{CHHCOCHH}), 2.52(1 \mathrm{H}, \mathrm{ddd}, J=14.6,3.8,1.4 \mathrm{~Hz}, \mathrm{CHHCOCH})$, 2.43-2.28 (4H, m, CHHCOCHH, $\left.\mathrm{CH}_{2} \mathrm{CHCH}\right), 1.91-1.62\left(2 \mathrm{H}, \mathrm{m}, \mathrm{OCHCH}_{2}\right) ; \delta \mathrm{c}\left(75.5 \mathrm{MHz} ; \mathrm{CDCl}_{3}\right)$; 206.5, 138.3, 132.2, 130.3, 128.4, 127.7, 127.6, 73.0, 72.9, 70.5, 69.2, 60.3, 47.6, 44.9, 37.5, 32.9.

\subsection{Synthesis of (2S,6S)-2-Dec-1-enyl-6-phenyltetrahydropyran-4-one (18)}

Potassium decenyl trifluoroborate salt $(0.057 \mathrm{~g}, 0.23 \mathrm{mmol})$ was reacted with $(S)$-2-phenyl-2,3dihydropyran-4-one $((S)-3)(0.020 \mathrm{~g}, 0.115 \mathrm{mmol})$ under the standard conditions. The crude residue was purified by flash column chromatography on silica gel (eluting with petrol:ethylacetate $9: 1$ ) to afford the title compound as a yellow oil ( $0.027 \mathrm{~g}, 72 \%$ yield $)$.

$\mathrm{R}_{\mathrm{f}}$ (petrol:ethyl acetate, 4:1); 0.69; $v_{\max }\left(\mathrm{CH}_{2} \mathrm{Cl}_{2}\right) / \mathrm{cm}^{-1} ; 3037,2924,2854(\mathrm{C}-\mathrm{H}), 1720(\mathrm{C}=\mathrm{O}), 1667$ $(\mathrm{C}=\mathrm{C}), 1603\left(\mathrm{C}=\mathrm{C}\right.$ aryl), 1249, $1052(\mathrm{C}-\mathrm{O}) ; \delta_{\mathrm{H}}\left(300 \mathrm{MHz} ; \mathrm{CDCl}_{3}\right) ; 7.38-7.29(5 \mathrm{H}, \mathrm{m} \mathrm{ArH}), 7.00(1 \mathrm{H}$, $\left.\mathrm{dt}, J=15.7,6.3 \mathrm{~Hz}, \mathrm{CH}_{2} \mathrm{CH}\right), 5.57\left(1 \mathrm{H}, \mathrm{dd}, J=15.7,4.9 \mathrm{~Hz}, \mathrm{CH}_{2} \mathrm{CHCH}\right), 5.11(1 \mathrm{H}, \mathrm{dd}, J=7.4 \mathrm{~Hz}, 5.4$ $\mathrm{Hz}, \operatorname{ArCHO}, 5.71(1 \mathrm{H}, \mathrm{dd}, J=9.6,4.7 \mathrm{~Hz}, \mathrm{CHCHO}), 2.74(1 \mathrm{H}, \mathrm{dd}, J=14.4,5.9 \mathrm{~Hz}, \mathrm{CHHCOCHH})$, $2.70(2 \mathrm{H}, \mathrm{d}, J=6.6 \mathrm{~Hz}, \mathrm{CH} H \mathrm{COCH}), 2.60(1 \mathrm{H}, \mathrm{dd}, J=14.4,4.6 \mathrm{~Hz}, \mathrm{CHHCOCH}), 2.06(2 \mathrm{H}, \mathrm{q}$, 
$\left.J=6.9 \mathrm{~Hz}, \mathrm{CH}_{2} \mathrm{CH}\right), 1.35\left(2 \mathrm{H}, \mathrm{dd}, J=13.0,5.9 \mathrm{~Hz}, \mathrm{CH}_{2} \mathrm{CH}_{2} \mathrm{CH}\right), 1.30-1.22\left(10 \mathrm{H}, \mathrm{m}, \mathrm{CH}_{3}\left(\mathrm{CH}_{2}\right) 8\right)$, $0.87\left(3 \mathrm{H}, \mathrm{t}, J=6.6 \mathrm{~Hz}, \mathrm{CH}_{3}\right) ; \delta_{\mathrm{C}}\left(75.5 \mathrm{MHz} ; \mathrm{CDCl}_{3}\right) ; 206.9,140.0,136.0,128.8,128.3,128.1,126.5$, 73.1, 72.9, 48.0, 45.4, 32.5, 31.9, 29.5, 29.4, 29.2, 29.0, 22.8, 14.2; HPLC (Chiralcel ODH: 98:2 Hexanes:propan-2-ol, $1.0 \mathrm{~mL} \cdot \mathrm{min}^{-1}, \mathrm{t}_{\mathrm{R}}=19.43 \mathrm{~min}$ (minor) and $21.42 \mathrm{~min}$ (major).

\subsection{Synthesis of (2S,6S)-2-(4-Bromophenyl)-6-phenyltetrahydropyran-4-one (19)}

4-Bromophenylboronic acid $(0.058 \mathrm{~g}, 0.287 \mathrm{mmol})$ was reacted with $(S)$-2-phenyl-2,3-dihydropyran4-one ((S)-3) (0.025 g, $0.144 \mathrm{mmol})$ under the standard conditions. The crude residue was purified by flash column chromatography on silica gel (eluting with petrol:ethylacetate 9:1) to afford the title compound as a colourless oil ( $0.044 \mathrm{~g}, 93 \%$ yield $)$.

$\mathrm{R}_{\mathrm{f}}$ (petrol:ethyl acetate, 4:1); 0.61; $\nu_{\max }\left(\right.$ neat) $/ \mathrm{cm}^{-1} ; 2983,2896(\mathrm{C}-\mathrm{H}), 1719(\mathrm{C}=\mathrm{O}), 1596,1494(\mathrm{C}=\mathrm{C}$ aryl), 1245, 1231 (C-O); $\delta_{\mathrm{H}}\left(300 \mathrm{MHz} ; \mathrm{CDCl}_{3}\right) ; 7.41(2 \mathrm{H}, \mathrm{d}, J=7.9 \mathrm{~Hz}, \mathrm{ArH}), 7.30-7.24$ (5H, m, $\left.\mathrm{ArH}\right)$, $7.17(2 \mathrm{H}, \mathrm{d}, J=8.3 \mathrm{~Hz}, \operatorname{Ar} H), 5.04(1 \mathrm{H}, \mathrm{t}, J=5.8 \mathrm{~Hz}, \mathrm{ArCHO}), 4.98(1 \mathrm{H}, \mathrm{t}, J=5.9 \mathrm{~Hz}, \operatorname{ArCHO}), 2.85$ $(1 \mathrm{H}, \mathrm{dd}, J=14.8,6.5 \mathrm{~Hz}, \mathrm{CHHCOCHH}), 2.76(1 \mathrm{H}, \mathrm{dd}, J=14.8,5.8 \mathrm{~Hz}, \mathrm{CHHCOCHH}), 2.75(2 \mathrm{H}$, $J=6.8 \mathrm{~Hz}, \mathrm{CH} H \mathrm{COCHH}) ; \delta_{\mathrm{C}}\left(75.5 \mathrm{MHz} ; \mathrm{CDCl}_{3}\right) ; 206.3,139.6,139.0,131.9,128.8,128.5,128.3$, 126.8, 122.2, 73.8, 72.9, 46.9, 46.3; HRMS $\left(\mathrm{ESI}^{+}\right)$calcd for $\mathrm{C}_{17} \mathrm{H}_{15} \mathrm{BrNaO}_{2}[\mathrm{M}+\mathrm{Na}]^{+} \mathrm{m} / z 353.0153$ found: $m / z$ 252.0124; HPLC (Chiralcel OJ, 9:1 Hexanes:propan-2-ol, $1.0 \mathrm{~mL} \cdot \mathrm{min}^{-1}, \mathrm{t}_{\mathrm{R}}=20.36 \mathrm{~min}$ (minor) and $28.61 \mathrm{~min}$ (major).

\section{Conclusions}

In summary, the catalytic conjugate addition of both aryl- and alkenylboronates to dihydropyranone templates have been accomplished in high yields, leading to the selective synthesis of 2,6-trans-tetrahydropyran derivatives. The selective formation of the 2,6-trans-tetrahydropyran stereoisomer is consistent with a mechanism involving alkene association and carbometalation on the less hindered face of the dihydropyranone. This methodology has simultaneously expanded the limited precedent for metal-catalysed addition of organoboron reagents to enantioenriched substrates and demonstrated the utility of sequential catalysis in the construction of "natural product-like" molecules.

\section{Acknowledgments}

We are grateful to the University of Bath and Atlas Genetics (http://www.atlasgenetics.com) for funding. We acknowledge the valuable assistance of Anneke Lubben (Mass Spectrometry) and John Lowe (NMR).

\section{Author Contributions}

H. J. Edwards and S. Goggins performed the research and C. G. Frost wrote the manuscript. All authors have approved the final content of the manuscript.

\section{Conflicts of Interest}

The authors declare no conflict of interest. 


\section{References}

1. Tian, P.; Dong, H.-Q.; Lin, G.-Q. Rhodium-catalyzed asymmetric arylation. ACS Catal. 2012, 2, 95-119.

2. Berthon, G.; Hayashi, T. Rhodium- and palladium-catalyzed asymmetric conjugate additions. In Catalytic Asymmetric Conjugate Reactions; Cordova, A., Ed.; Wiley-VCH: Weinheim, Germany, 2010; Chapter 1, pp. 1-70.

3. Edwards, H.J.; Hargrave, J.D.; Penrose, S.D.; Frost, C.G. Synthetic applications of rhodium catalysed conjugate addition. Chem. Soc. Rev. 2010, 39, 2093-2105.

4. Hargrave, J.D.; Allen, J.C.; Frost, C.G. Alternatives to organoboron reagents in rhodium-catalyzed conjugate additions. Chem. Asian J. 2010, 5, 386-396.

5. Hayashi, T.; Yamasaki, K. Rhodium-catalyzed asymmetric 1,4-addition and its related asymmetric reactions. Chem. Rev. 2003, 103, 2829-2844.

6. Fagnou, K.; Lautens, M. Rhodium-catalyzed carbon-carbon bond forming reactions of organometallic compounds. Chem. Rev. 2003, 103, 169-196.

7. Hayashi, T.; Takahashi, M.; Takaya, Y.; Ogasawara, M. Catalytic cycle of rhodium-catalyzed asymmetric 1,4-addition of organoboronic acids. Arylrhodium, oxa- $\pi$-allylrhodium, and hydroxorhodium intermediates $J$. Am. Chem. Soc. 2002, 124, 5052-5058.

8. Filloux, C.M.; Rovis, T. Rh(I)-bisphosphine-catalyzed asymmetric, intermolecular hydroheteroarylation of $\alpha$-substituted acrylate derivatives. J. Am. Chem. Soc. 2015, 137, 508-517.

9. Navarre, L.; Martinez, R.; Genet, J.; Darses, S. Access to enantioenriched $\alpha$-amino esters via rhodium-catalyzed 1,4-addition/enantioselective protonation. J. Am. Chem. Soc. 2008, 130, 6159-6169.

10. Moss, R.J.; Wadsworth, K.J.; Chapman, C.J.; Frost, C.G. Rhodium-catalysed tandem conjugate addition-protonation: An enantioselective synthesis of 2 -substituted succinic esters. Chem. Commun. 2004, 1984-1985.

11. Ramnauth, J.; Poulin, O.; Bratovanov, S.S.; Rakhit, S.; Maddaford, S.P. Stereoselective C-glycoside formation by a rhodium(I)-catalyzed 1,4-addition of arylboronic acids to acetylated enones derived from glycals. Org. Lett. 2001, 3, 2571-2573.

12. Zoute, L.; Kociok-Kohn, G.; Frost, C.G. Rhodium-catalyzed 1,4-additions to enantiopure acceptors: Asymmetric synthesis of functionalized pyrrolizidinones. Org. Lett. 2009, 11, 2491-2494.

13. Hargrave, J.D.; Bish, G.; Frost, C.G. Switching stereoselectivity in rhodium-catalysed 1,4-additions: The asymmetric synthesis of 2-substituted pyrrolizidinones. Chem. Commun. 2006, 4389-4391.

14. Clarke, P.A.; Santos, S. Strategies for the formation of tetrahydropyran rings in the synthesis of natural products. Eur. J. Org. Chem. 2006, 9, 2045-2053.

15. Pellissier, H. Asymmetric hetero-Diels-Alder reactions of carbonyl compounds. Tetrahedron 2009, 65, 2839-2877.

16. Tietze, L.F.; Kettschau, G.; Gewert, J.A.; Schuffenhauer, A. Hetero-Diels-Alder reactions of 1-oxa-1,3-butadienes. Curr. Org. Chem. 1998, 2, 19-62.

17. Kumaraswamy, G.; Ramakrishna, G.; Naresh, P.; Jagadeesh, B.; Sridhar, B. A flexible enantioselective total synthesis of Diospongins A and B using catalytic hetero-Diels-Alder/Rh-catalyzed 1,4-addition and asymmetric transfer hydrogenation reactions as key steps. J. Org. Chem. 2009, 74, 8468-8471. 
18. Danishefsky, S.; Kerwin, J.F.; Kobayashi, S. Lewis acid catalyzed cyclocondensations of functionalized dienes with aldehydes. J. Am. Chem. Soc. 1982, 104, 358-360.

19. Frost, C.G.; Penrose, S.D.; Gleave, R. Rhodium catalysed conjugate addition of a chiral alkenyltrifluoroborate salt: The enantioselective synthesis of hermitamides A and B. Org. Biomol. Chem. 2008, 6, 4340-4347.

20. Lennox, A.J.J.; Lloyd-Jones, G.C. Organotrifluoroborate hydrolysis: Boronic acid release mechanism and an acid-base paradox in cross-coupling. J. Am. Chem. Soc. 2012, 134, 7431-7441.

21. Jørgensen, K.A. Catalytic asymmetric hetero-Diels-Alder reactions of carbonyl compounds and imines. Angew. Chem. Int. Ed. 2000, 39, 3558-3588.

22. Wang, B.; Feng, X.; Huang, Y.; Liu, H.; Cui, X.; Jiang, Y. A highly enantioselective hetero-Diels-Alder reaction of aldehydes with Danishefsky's Diene catalyzed by chiral titanium(IV) 5,5',6,6',7,7',8,8'-octahydro-1,1'-bi-2-naphthol complexes. J. Org. Chem. 2002, 67, 2175-2182.

23. Smith, A.B., III; Fox, R.J.; Vanecko, J.A. (+)-Sorangicin a synthetic studies. Construction of the C(1-15) and C(16-29) subtargets. Org. Lett. 2005, 7, 3099-3102.

Sample Availability: Samples of the compounds are not available from the authors.

(C) 2015 by the authors; licensee MDPI, Basel, Switzerland. This article is an open access article distributed under the terms and conditions of the Creative Commons Attribution license (http://creativecommons.org/licenses/by/4.0/). 